\title{
Impact of Changing Lifestyle on Endometriosis Related Pain
}

\author{
Gehan Ebrahim Ghonemy ${ }^{1}$ and Nadia Bassouni El Sharkawy ${ }^{2}$ \\ ${ }^{I}$ Assistant professor, ${ }^{2}$ Lecturer of Maternal and Newborn Health Nursing Department, Faculty of Nursing, \\ Cairo University, Cairo, Egypt.
}

\begin{abstract}
Endometriosis is a condition or disease, when endometrial tissue, that normally lines the inner surface of the body of the uterus, is found elsewhere. This study aimed to evaluate the impact of changing lifestyle on endometriosis related pain, using one group pre-post test quasi experimental time series research design. Subjects and methods: A purposive sample of 50 women diagnosed with endometriosis was selected from gynecology inpatient departments \& outpatient clinic at El Galaa Maternity Hospital. The data collection tools were Sociodemographic \& Knowledge Assessment Sheet and Visual Analog Scale (VAS). A procedure were carried out through three phases as: interviewing, implementation, and evaluation phase. Results of the study revealed a statistically significant decrease severity of pain three months post education compared to preeducation $p<0.00$.There were statistically significant difference between mean total knowledge score at per-and post teaching $p<0.001$.There was only a statistically positive correlation between pre-education type of diet with symptoms of endometriosis ( $r=0.334, P=0.015)$. While no correlation between three moths post education exercise and symptoms of endometriosis. The study concluded that health education about changing lifestyle particularly dietary health education for women with endometriosis have shown beneficial improvement of knowledge and reduction endometriosis related pain. The study recommended that adoption of healthy lifestyle is important and beneficial for women with endometriosis. A simplified and comprehensive booklet should be available for all patients with endometriosis, this booklet should include a clear, brief and simple explanation about non pharmacological modalities to reduce endometriosis related pain
\end{abstract}

Key Words: lifestyle changes, Health education endometriosis, pain

\section{Introduction}

Endometriosis is a condition or disease, when endometrial tissue, that normally lines the inner surface of the body of the uterus, is found elsewhere. Similarly to the eutopic endometrium, the ectopic endometrial tissue is under the influence of hormones, proliferates changes in histological characteristics undergoes degeneration and then shedding, just like in the case of menstruation. During these processes it might cause severe destruction of the surrounding tissues and organs[1] It is estrogen-dependent chronic inflammatory disease and often progressive, resulting in worsening pain and other symptoms over time [2,3]. Endometriosis affects an estimated 1 in 10 women during their reproductive years (usually between the ages of 15 to 49), which is approximately 176 million women worldwide. However, endometriosis can start as early as a girl's first period and the menopause may not resolve the symptoms of endometriosis, especially if the woman has scar tissue or adhesions from the disease and/or surgery. It can severely alter quality of life and leads to extensive problems with fertility and loss of work time [4,5].

Endometriosis might remain asymptomatic and discovered accidentally. However, it may cause symptoms as painful menstruation (dysmenorrhea), painful intercourse (dyspareunia), painful micturition (dysuria), painful defecation (dyschezia), lower back or abdominal discomfort, chronic pelvic pain (non-cyclic abdominal and pelvic pain of at least 6 months' duration), cyclic rectal bleeding or hematuria (bowel or bladder invasion), and cyclic dyspnea secondary to catamenial pneumothorax, susceptibility to development of adenocarcinoma and bleeding into the surrounding tissues, resulting in inflammation and formation of scarring and adhesions. It is peculiar, that symptom severity does not correlate well with the extent or progression of the lesions [6,7]. Endometriosis etiology is still unclear, but retrograde menstruation is widely accepted as a contributing factor to the disease. Other suggested main risk factors is failure of immune mechanisms to destroy the ectopic tissue and abnormal differentiation of endometriotic tissue has been proposed as underlying mechanisms in a stromal-cell defect associated with increased estrogen and prostaglandin production, along with resistance to progesterone $[8,9,10]$.

Since there is no cure, medical treatments must be effective and safe to use until the age of menopause or until pregnancy is desired [3]. So, one theory is that dietary fat influences female body's production of prostaglandins, chemicals that stimulate uterine contractions and affect ovarian functioning. It's thought that high levels of prostaglandins could lead to higher production of estrogen, which could influence the growth of endometrial tissue. Other studies find a link between high-fat diets and levels of circulating estrogen; the more fat in diet, the more estrogen female body produces. This also occurs if women are overweight, and women more likely to be overweight if she follows a diet high in red meat and low in fruits and vegetables [7]. 
Unfortunately, few studies on endometriosis and lifestyle focus on whether certain diets or levels of activity are connected to endometriosis symptoms, not whether those aspects improve endometriosis-related symptoms. Diets deficient in nutrients results in changes in lipid metabolism, oxidative stress and promote epigenetic abnormalities, that may be involved in the genesis and progression of the disease. Foods rich in omega3 with anti-inflammatory effects, supplementation with $\mathrm{N}$-acetylcysteine, vitamin $\mathrm{D}$ and resveratrol, in addition to the increased consumption of fruits, vegetables (preferably organic) and whole grains exert a protective effect, reducing the risk of development and possible regression of disease [11].

Moving on to exercise or physical activities as another aspect of lifestyle, regular physical exercise seems to have protective effects against diseases that involve inflammatory processes since it induces an increase in the systemic levels of cytokines with anti-inflammatory and antioxidant properties and also acts by reducing estrogen levels. Evidence has suggested that the symptoms associated with endometriosis result from a local inflammatory peritoneal reaction caused by ectopic endometrial implants [12].

Also, its known that women who exercise intensely tend to have lighter periods with reduced ovarian stimulation and estrogen production. In one study, researchers evaluated the effects of high-intensity physical activity on a woman's risk of endometriosis. They found that women who averaged 2.5 hours of high-intensity activity (jogging, bicycling or aerobics) were $63 \%$ less likely to have endometriosis [13]. In the scope of how these exercises affect the women health in general \& endometriosis in a specific, its known that exercise releases endorphins. When exercise carried out, the brain releases "feel good" chemicals called endorphins. These naturally occurring hormones work like pain relievers to lower pain. Regular exercise lowers the amount of estrogen in the body which the goal of endometriosis treatment is to lower estrogen levels to improve endometriosis symptoms [14].

Therefore, with the literature available it is not possible to point out the real role of physical exercise in endometriosis. Thus, until now there is a speculation about this topic. In this respect, its believed that studies well controlled, using validated instruments for evaluation and follow up, well- defined study groups and well established exercise protocol can demonstrate the real role of physical exercise on treatment of endometriosis. On this basis, experimental models of endometriosis would be justified because they would permit the characterization of the time course of the disease in order to elucidate whether physical exercise is indeed able to interfere with the development of the endometriosis injury. In addition, it would be possible to determine what intensity would be necessary for physical exercise to be used in both a preventive and curative manner regarding the disease [15]. Reducing the amount of red meat in diet, upping intake of fresh fruit and vegetables and getting three or more days of moderate- to high-high intensity exercise will help in several ways and may make a difference in the severity of endometriosis related pain. If any female diagnosed with endometriosis, she may be wondering if there is anything she can do to feel better besides taking medication. Healthy nutrition and exercise play an important role in maintaining overall health [14]. Nurses are committed to assist and support the patients to make lifestyle changes that improve the patient's health and wellbeing. Nurses have an essential role in health promotion through disease management by providing support and much needed information to the patient with endometriosis. In addition, this will facilitate quality of care to improve quality of life, reduce pain, and prevent further progression of disease. Nurses also can play that role of health teaching to help women with endometriosis to cope with her condition throughout support women and her family to adapt with this condition and provide source of follow up.

\subsection{Significance of the Study}

Worldwide, the prevalence of endometriosis ranges from $6 \%-10 \%$, the prevalence is reported in $20 \%$ $90 \%$ of women suffering from pelvic pain or infertility [7,16]. While, in Egypt the prevalence of endometriosis is difficult to determine because of lack of documentation or filling system for cases of endometriosis and the only reliable diagnostic test is laparoscopy. Disease prevalence in Egypt started to increase as observed through clinical practice at El Galaa Maternity Hospital and other private clinics. Health care team should be involved in providing health education about diet \& exercise that can interfering with endometriosis pain. From the researchers point of view, there are lack of evidence about published studies in Egypt about the importance of health education related to changing lifestyle that could affect endometriosis related pain, and indirectly improving woman's life and work productivity so, this study will help the researchers to explore critical areas in the management of endometriosis that may not discovered before, and a new modality for the management of endometriosis related pain that may be conducted from a nursing perspective. $+$

\subsection{Operational Definition:}

1.1.1.Lifestyle: In the present study, it was referred to two variables only; the dietary habits \& exercise practices throughout three months follow up and health education sessions. 
1.1.2.Endometriosis Related Pain: In the present study, pain is caused by inflammatory reaction and shedding of endometrial cells in the form of dysmenorrhea, dyspareunia, dysuria, painful defecation (dyschezia), lower back or abdominal discomfort and chronic pelvic pain (non-cyclic abdominal and pelvic pain ), that is also called endometriosis symptoms.

\subsection{Aim of the study:}

The present study aimed to evaluate the impact of changing lifestyle on endometriosis related pain.

\subsection{Research Hypothesis:}

Women who will change their lifestyle (follow dietary \& exercises health education) will have lesser post-mean pain score than pre-mean pain score.

\section{Subjects And Methods}

\subsection{Research Design:}

One group pre-posttest quasi experimental time series research design was adopted in this study to achieve the study's aim.

\subsection{Setting:}

The study was conducted at gynecology Inpatient departments \& outpatient clinic of El Galaa Maternity Hospital.

\subsection{Sample:}

A purposive sample of 50 women diagnosed with endometriosis. Sample size was calculated based on a power analysis of $0.95(\beta=1-0.95=0.5)$ at alpha .05 (one -sided) with large effect size (0.5) was used as the significance. The sample was recruited according to the following inclusion criteria; women with different stages of endometriosis during their reproductive years even single or married after diagnostic laparoscopy for endometriosis, under management of endometriosis related pain, and free from any other chronic medical disorder.

\subsection{Tools for Data Collection:}

Two tools were used and filled by researchers to collect the required data for this research

Tool (I): Sociodemographic and Knowledge Assessment Sheet: it was developed by researchers and consisted of three parts.

Part (1): Sociodemographic data including such as age, residence, income, marital status, educational level, occupation, etc.

Part (2): Obstetrical data including obstetrical \& gynecological history; and data related to history of endometriosis, how it was discovered, duration of disease and management modality women receive .

Part (3): 8Questions to assess patient's knowledge and practices related to endometriosis such as definition, its common symptoms; lifestyle assessment (diet and exercise) as types of food that can affects endometriosis pain, and type of exercise that can positively or negatively affect endometriosis related pain.

\section{Scoring system}

Each item in the sheet was given a score of three marks for complete correct answer, two marks for incomplete correct answer, one mark for incorrect answer, and zero for missed answer. Then all scores summed up and illustrated into three categories: a score range from $75 \%$ to $100 \%$ (from 38 to 51 ) illustrated that patients have good knowledge; while a score from $50 \%$ to less than $75 \%$ (from 26 to less than 38 ) illustrated satisfactory level and a score less than $50 \%$ (less than 26) illustrated that patients have poor knowledge.

\section{Tool (II).Visual Analogue Pain Scale (VAS).}

It was adopted from [17] and used by the researchers to assess the subject's level of pain intensity. VAS consists of a straight line with the endpoints defining extreme limits such as 'no pain at all' and 'pain as bad as it could be'. The measurement was from zero to 10, in which zero mean no pain while 10 illustrate worst pain. The subject verbally select a value that is most in line with the intensity of pain that they have experienced in the last 24 hours. VAS Rating Pain level from 0 to 10, Level (0) denoted no Pain, level from 1 to 3 denoted mild pain, a score from 4 to 6 denoted moderate pain and score from 7 to 10 indicated worst or severe pain. A high reliability was proved by a strong correlation with a coefficient of $0.976(\mathrm{p}<0.001)$. A high internal consistency of the VAS score was shown by a Cronbach-alpha of 0.9117. VAS Rating Pain level from 0 to 10 , Level ( 0$)$ No Pain , (1-3) Mild Pain, (4-6 ) Moderate Pain, and (7-10) Severe Pain [18]. 


\subsection{Tool Validity:}

Tools for data collection submitted to 3 nursing experts in the field of Maternal and Newborn Health Nursing and 2 Gynecologists in the field of Obstetrics and Gynecology to test the content validity, modifications were carried out according to the experts judgment on clarity of sentences and the appropriateness of contents.

\subsection{Pilot study:}

A pilot study was conducted on $10 \%$ of the studied women to assess the clarity, feasibility, and objectivity of the tools. Modification was carried out based on the results of the pilot. Women participated in the pilot study were excluded from the sample, because of certain correction was carried out on the tool after piloting.

\subsection{Ethical Consideration:}

An official permission was granted from the administrative personnel El Galaa hospital. The researchers explained the aim of the study to the women and informed them that the information obtained will be confidential and their participation was in a voluntary base. A written Informed consent was taken from each woman to obtain their acceptance to participate in the study.

\subsection{Procedure:}

Data were collected through a period of six months from first of July 2016 till the end of December 2016. Data were collected through three phases: interviewing \& assessment, implementation, and evaluation phase.

- Interviewing \& Assessment phase: Interviewing was carried out using structured interviewing questionnaire and pretest women knowledge and disease symptoms assessment. The researchers identified themselves to the women and explained the study aim, benefits and the procedures to be performed. Women who were willing to participate in the study and met the inclusion criteria were approached by the researchers. Base line assessment of patient's sociodemographic, medical \& obstetrical data and knowledge about endometriosis was performed using tool (I) \& these was carried out before the women had their menses started, using face to face interviewing session in order to plan for the next meeting, as well as trained women about how they use the VAS during their coming menstruation. Also, women were assessed for level of pain using tool (II) by using telephone call as most of the women refused to meet the researcher at that time. The time needed for completing the questionnaire was 20 minutes for each woman \& VAS one min.

- Implementation phase: In this phase, the health education was provided for women during their first meeting during hospitalization hours or follow up at outpatient gynecological clinic. An oral instruction as a method of teaching supported with booklet was used using Arabic understandable language. There was a total of 3 sessions each one for 30 minutes. Each educational session had a maximum 3-5 patients, \& sometimes carried out individually especially during the second one. At the start of the session each patient obtained a copy of a simplified booklet that included information about disease \& ways of managements of its pain. In the first session, the researchers started to establish rapport with patient, then verbal instructions about disease process as definition, signs \& symptoms, diagnosis \& treatment modalities. Measures that important to reduce the disease related pain was introduced as diet that could affect endometriosis related pain, advice women to start eating healthy diet which rich in fibers (fruits \& vegetables) and healthy ways of cooked meals (boiled and grilled) and to increase diet that has anti-inflammatory and antioxidant actions like diet rich in Omga3. As well as education about negative impact of high salt, caffeine, fried food and fatty red meat on endometriosis related pain. Also, an education related to type of exercises that could be used by women from 2-3 times per week for 20-30 minutes at least can be carried out through walking, or gymnastics, or aerobic, or bicycle, or running. During that session the researchers trained the women about how to mark their pain level on the line between the two endpoints on visual analogue scale and asked women to plot and rate their level of pain during its occurrence (e.g. during the menstruation , intercourse , defecation, etc.). In the second session one month later, the researchers met women to ensure that they follow researchers instructions as well as to respond for any questions. The third session was by the end of the third month. Women were checked for acquisition of knowledge as well as to determine their improvement using VAS. Each patient was contacted at least one time / 2-3 weeks for about 3 months through telephone or direct contact in outpatient gynecological clinics during follow up to reinforce provided knowledge and respond to their questions if any. Sometimes women refused to come \& prefer to used phone call because of their fatigue condition. Certain women call for discussing certain issues related to that teaching content \& they received their need using phone answered from the researchers

- Evaluation phase: Each women was evaluated two times: The first evaluation is in the interviewing and assessment phase using tools I and II. The second evaluation was done 3 month post implementation of the 
health education sessions. All subjects were assessed for their knowledge, their lifestyle changes (diet and exercise) related to endometriosis, and level of endometriosis related pain.

\subsection{Statistical design:}

Data was tabulated and analyzed, all statistical calculations were done using computer session SPSS (Statistical Package for the Social Science; SPSS version 21). Descriptive statistics : data were presented in the form of mean \pm standard deviation or frequencies and percentages. Inferential statistics: Paired $(t)$ test was used to determine the difference between pretest and post-test evaluations of the same group . Chi-square test $\left(\chi^{2}\right)$ : was used to study association between two qualitative variables. Correlation between different variables were done using Pearson moment correlation. $P$ values less than 0.05 will considered statistically significant.

\section{Results}

Women's age ranged between 19-39 years old with mean of 29.8 4 4.02. Sixty nine percent of them was married. More than three quarters $(76 \%)$ of the women had completed technical secondary school education \& $20 \%$ highly educated, and $72 \%$ were housewives. Sixty eight percent of women live in urban. Four percent (2) of women were single. Monthly income was more than one thousand in $74 \%$ of the study sample (table 1).

Findings of this research indicated that 73\% (35/48) of women were nullipara, 27\% (13/48) had history of delivery; $10.4 \%$ of them had history vaginal delivery and $16.6 \%$ had history of cesarean section. Thirty one point twenty five percent of women had previous history of abortion. Regarding gynecological diseases, $44 \%$ of women had gynecological problems as polycystic ovaries, toxoplasmosis fetalis, chocolate cyst, \& vaginal infection (18\%, $8 \%, 6 \%, \& 12 \%$ respectively). Findings indicated that more than one third $(44 \%)$ of women had previous gynecological operations as ovarian cyst removal, Dilatation and Curettage, and hysteroscopy $(18 \%, 22 \%$, and $14 \%$ respectively). Regarding stages of endometriosis, $44 \%$ of women had a third stage of endometriosis followed by $40 \%$ in second stage. While $12 \%$ complains of first stage endometriosis and $4 \%$ had fourth stage endometriosis. The results showed that $90 \%$ of women didn't receive any dietary supplementations while $10 \%$ received vitamin $\mathrm{D}, \mathrm{C}$, and $\mathrm{E}$ in their diet by (4\%,2\%, $2 \%$ respectively).

Endometriosis symptoms reported by women were dysmenorrhea, non cyclic pelvic pain, dyspareunia, painful defecation, and painful urination $(48 \%, 26 \%, 18 \%, 6 \%, \& 2 \%$ respectively) Fig (1). Surgical ablation was done for $50 \%$ of women as a management modality and another half of the sample not receives any kind of management

More than half (62\%) of the sample was depended on red meat in their meals and fried foods (56\%). While three months post health education more than half $(58 \%)$ of the sample changed their dietary habits to depend on fruits and green vegetables in their meals with equal percentage of $48 \%$ of the sample was preferred boiling and grilling their foods while they cooking. There was no statistically significant difference regarding to type of diet and way of cooking pre an three months post education $\left(\chi^{2}=13.423, \mathrm{P}=0.144 \& \chi^{2}=4.102, \mathrm{P}=0.905\right.$ respectively) (table 2 ).

The results indicated that only $10 \%$ (5) of women practice exercises pre education compared to $20 \%$ (10) after three months from health education sessions, as most of the women did not like to use exercises because they afraid from becoming pregnant at any times \& they believe that they can loss her pregnancy by using any kind of exercises. Most of the study women who follow the exercise pre-post education were (6\% \& 8\% respectively) prefer to walk for thirty (30) minutes rather than using gymnastic or running.

Table 3 showed that the commonest symptom was dysmenorrhea (48\%) which reflect almost half of women; $30 \%$ of them depend on meat as main dietary habit. Twenty six percent had a non cyclic pelvic pain ; 18\% of them also depend on meat as main dietary habit with statistically significant association between type of diet and endometriosis symptoms $\left(\chi^{2}=23.501, \mathrm{P}=0.02\right)$.

There were statistically significant difference between mean of total knowledge score at pre- and post teaching $(t=-21.664, \mathrm{P}<0.001)$ and this was clear in the post test as $100 \%$ of women had good level of knowledge three months after health education (table 4$)$.

Table (5) presented total pain score pre and post education among women with endometriosis. It was showed that the majority of them had severe pain at pre- education (74\%). While at post education more than half of women had moderate pain $(60 \%)$ with highly statistically significant difference between mean pain score pre and post education using VAS $(7.44 \pm 1.45 \& 5.7 \pm 1.34$ respectively) (t-test=10.798, $<<0.001)$.

There was only a statistically positive correlation between pre- education type of diet with symptoms of endometriosis $(\mathrm{r}=0.334, \mathrm{P}=0.015)$. While there were no correlation between pre- and post education type of diet $(\mathrm{r}=0.064, \mathrm{P}=0.0658)$ as well as no correlation was found between post-education type of diet with symptoms of endometriosis $(\mathrm{r}=-0.150, \mathrm{P}=0.299)$. No correlation was found between post education exercise and women's symptoms of endometriosis $(\mathrm{r}=0.052, \mathrm{p}=0.87$ ) (table 6$)$. 
Table 1 Distribution of the Sample by Their Sociodemographic Characteristics $(n=50)$

\begin{tabular}{|c|c|c|}
\hline Socio demographic Characteristics & No. & $\%$ \\
\hline \multicolumn{3}{|l|}{ Age : mean $29.8 \pm 4.02$} \\
\hline$<20$ & 1 & 2 \\
\hline $20-$ & 5 & 10 \\
\hline $25-$ & 20 & 40 \\
\hline $30-$ & 20 & 40 \\
\hline $35-40$ & 4 & 8 \\
\hline \multicolumn{3}{|l|}{ Educational level: } \\
\hline Primary school & 2 & 4 \\
\hline Secondary school & 38 & 76 \\
\hline University & 10 & 20 \\
\hline \multicolumn{3}{|l|}{ Marital status } \\
\hline Married & 48 & 96 \\
\hline Single & 2 & 4 \\
\hline \multicolumn{3}{|l|}{ Residence } \\
\hline Rural & 16 & 32 \\
\hline Urban & 34 & 68 \\
\hline \multicolumn{3}{|l|}{ Family monthly income } \\
\hline From 500 to 1000 & 13 & 26 \\
\hline More than 1000 & 37 & 74 \\
\hline
\end{tabular}

Figure 1 Distribution of the Sample by Their Symptoms of Endometriosis $(n=50)$

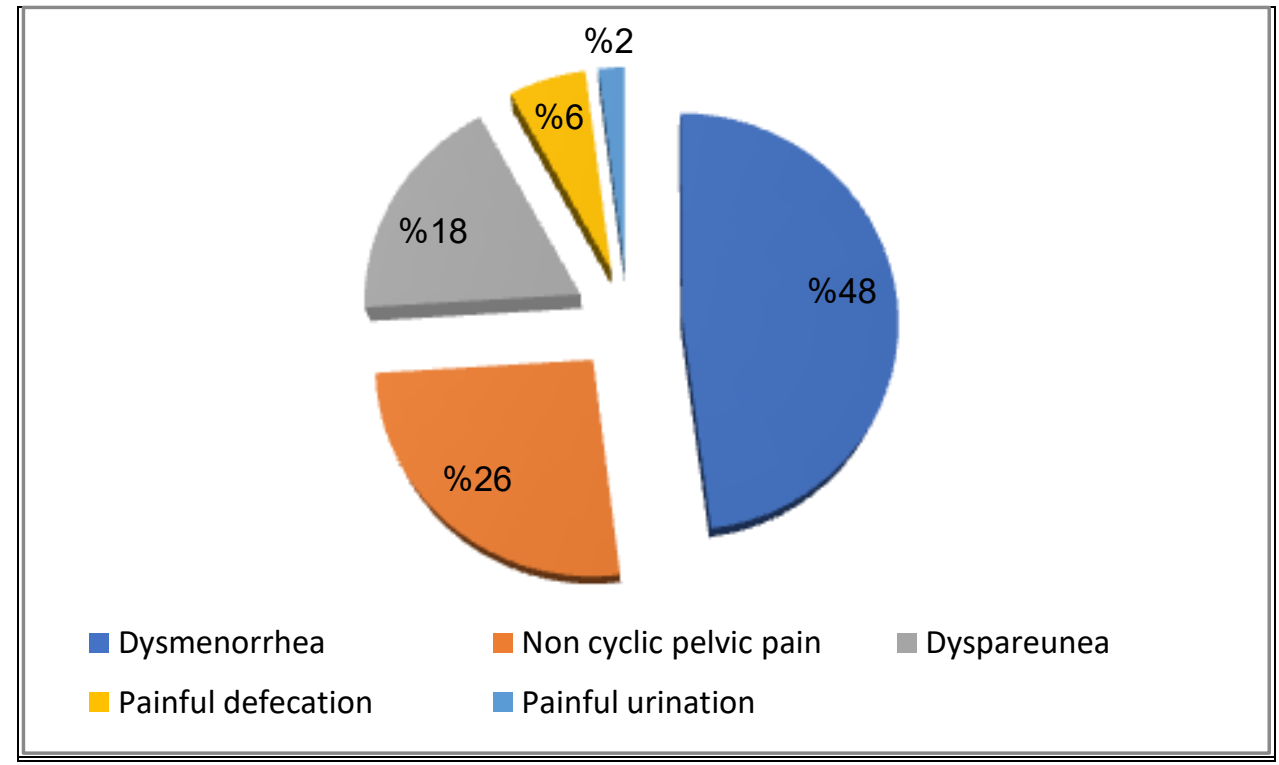

Table 2 Distribution of the Sample by Their Dietary Habits Pre and Three Months Post Health Education $(\mathrm{n}=50)$.

\begin{tabular}{|l|c|c|c|c|c|}
\hline Items & Pre health education & 3 months after education & Test \\
\hline Dietary habits & No & $\%$ & No & $\%$ & \multirow{2}{*}{$\chi^{2}=13.423$} \\
\hline Fruits and vegetables & 3 & 6.0 & 29 & 58.0 & 2.0 \\
$\mathrm{P}=0.144$ \\
\hline Red meat & 31 & 62.0 & 1 & 6 & \\
\hline Carbohydrate & 11 & 22.0 & 6 & 12.0 & \\
\hline Mixed & 5 & 10.0 & 14 & 28.0 & \\
\hline Way of cooking women prefer & No & $\%$ & No & $\%$ & \multirow{2}{*}{$\chi^{2}=4.102$} \\
\hline Boiled & 4 & 8.0 & 24 & 48.0 & \\
\hline Fried & 28 & 56.0 & 1 & 2.0 & \\
\hline Grilled & 3 & 6.0 & 24 & 48.0 & \\
\hline Foundry & 15 & 30.0 & 1 & 2.0 & \\
\hline
\end{tabular}

* significant at less than 0.05 level 
Impact of Changing Lifestyle on Endometriosis Related Pain

Table 3 Relationship Between Endometriosis Symptoms and Type of Diet Pre Health Education

\begin{tabular}{|c|c|c|c|c|c|c|c|c|c|c|c|}
\hline Variable & \multicolumn{8}{|c|}{ Type of Diet } & \multirow{2}{*}{\multicolumn{2}{|c|}{ Total }} & Test \\
\hline \multirow{2}{*}{ Symptoms } & \multicolumn{2}{|c|}{ Fruits and Vegetables } & \multicolumn{2}{|c|}{ Red Meat } & \multicolumn{2}{|c|}{ Carbohydrate } & \multicolumn{2}{|c|}{ Mixed } & & & \multirow{7}{*}{$\begin{array}{c}\chi^{2}=23.501 \\
\mathrm{P}=0.02\end{array}$} \\
\hline & No. & $\%$ & No. & $\%$ & No. & $\%$ & No. & $\%$ & No. & $\%$ & \\
\hline Dysmenorrhea & 2 & 4 & 15 & 30 & 1 & 12 & 6 & 2 & 24 & 48 & \\
\hline Non cyclic pelvic pain & 1 & 2 & 9 & 18 & 2 & 4 & 1 & 2 & 13 & 26 & \\
\hline Dyspareunia & 0 & 0.0 & 6 & 12 & 3 & 6 & 0 & 0.0 & 9 & 18 & \\
\hline Painful defecation & 0 & 0.0 & 1 & 2 & 0 & 0.0 & 2 & 4 & 3 & 6 & \\
\hline Painful urination & 0 & 0.0 & 0 & 0.0 & 0 & 0.0 & 1 & 2 & 1 & 2 & \\
\hline
\end{tabular}

Table 4 Women's Knowledge Assessment Pre and Post Education $(\mathrm{n}=50)$

\begin{tabular}{|c|c|c|c|c|c|}
\hline \multirow{2}{*}{ Knowledge Assessment } & \multicolumn{2}{|c|}{ Pre education } & \multicolumn{2}{|c|}{ Three Months } & \multirow{2}{*}{ Test } \\
\hline & No & $\%$ & No & $\%$ & \\
\hline Poor & 34 & 68.0 & 0 & 0.0 & \\
\hline Satisfactory & 10 & 20.0 & 0 & 0.0 & \\
\hline Good & 6 & 12.0 & 50 & 100 & \\
\hline Mean & \multicolumn{2}{|c|}{$23.52 \pm 8.86$} & \multicolumn{2}{|c|}{$50.82 \pm .388$} & $\begin{array}{c}t=-21.664 \\
P<0.001\end{array}$ \\
\hline
\end{tabular}

Table 5 Pain Scores Pre and Post Education of Women with Endometriosis $(n=50)$

\begin{tabular}{|l|c|c|c|c|c|}
\hline \multirow{2}{*}{ Pain level } & \multicolumn{2}{|c|}{ Pre education } & \multicolumn{2}{c|}{ Three Months } & \multirow{2}{*}{ Test } \\
\cline { 2 - 5 } & No & $\%$ & No & $\%$ & \\
\hline Mild & 0 & 0.0 & 7 & 8.0 & \\
\hline Moderate & 13 & 26.0 & 34 & 60.0 & \\
\hline Severe & 37 & 74.0 & 9 & 32.0 & \multirow{2}{t}{$\begin{array}{c}\mathrm{t}=10.798 \\
\mathrm{P}=<0.001\end{array}$} \\
\hline Mean & \multicolumn{2}{|c|}{$7.44 \pm 1.45$} & \multicolumn{2}{|c|}{$5.7 \pm 1.34$} & 0 \\
\hline
\end{tabular}

Table 6 Correlation Matrix Between Pre- Post Education Type of Diet, Post Education Exercise and Symptoms of Endometriosis $(n=50)$.

\begin{tabular}{|l|c|c|c|c|}
\hline \multirow{2}{*}{ Variables } & \multicolumn{3}{|c|}{ Pre- post education type of diet } & \multirow{2}{*}{ Post Education Exercise } \\
\cline { 2 - 5 } & Pre-type of diet & Post- type of diet & \\
\hline \multirow{2}{*}{ Pre-type of diet } & $\mathrm{R}$ & ------ & .064 & \\
\cline { 2 - 5 } & $\mathrm{P}$ & ------ & .658 & \\
\hline \multirow{2}{*}{ Post- type of diet } & $\mathrm{R}$ & .064 & ----- & \\
\cline { 2 - 5 } & $\mathrm{P}$ & .658 & ----- & 0.052 \\
\hline \multirow{2}{*}{ Symptoms of endometriosis } & $\mathrm{R}$ & .344 & $-.150-$ & 0.87 \\
\cline { 2 - 5 } & $\mathrm{P}$ & .015 & .299 & \\
\hline
\end{tabular}

* Correlation is significant at less than 0.05 level

\section{Discussion}

Endometriosis is a debilitating chronic disease that can affect many aspects of everyday life owing to symptoms such as pain and fatigue. Better understanding of the long term and wide ranging impact of endometriosis on women's lives at different life stages could benefit policy makers, health professionals and the lay population in reducing the negative impact of endometriosis and improving women's life experiences[19].Chronic pain was the root cause for study the impact of endometriosis experienced by women in this research. Education was interrupted, careers impeded and social participation curtailed because of the impact of symptoms on everyday functioning. Well-informed nurses and other health professionals can play an important role in mitigating this impact through proactively taking the time to discuss with women potential issues along with strategies to manage the disease.

The aim of the present study was to evaluate the impact of changing lifestyle on endometriosis related pain. As a women with endometriosis facing many problem to cope with their disease, so this study implementing a health education sessions to assist these women to cope with their discomfort through changing certain lifestyles which will positively affect their condition. One hypothesis was applied to be investigating into that study, this hypothesis predicted a decrease of mean pain scores among women who were exposed to this health education session (post mean pain score was lesser than pre-one) and this effect was followed throughout three months.

Findings of the present study indicated that more than two thirds $(73 \%)$ of women with endometriosis were nullipara. In agreement with the present findings, many clinical and epidemiological data suggested an inverse association between parity and the risk of developing endometriosis [20,21,22].

In the present study, the commonest symptoms were severe dysmenorrhea (48\%) was the most prominent symptom of women with endometriosis. Almost other studies results were in congruent with the present result, as in a systematic review based on 15 selected studies, the prevalence of visually confirmed endometriosis among girls with severe dysmenorrhea was 62\% [23] and $68.8 \%$ [24]. However the overall 
prevalence of endometriosis among the adolescent girls as reported by the American Society of Reproductive Medicine classified moderate - severe endometriosis was 32\% [23].This disparity of incidence can be explained by the population studied, the sample size used, and the method of diagnosis whether clinical or surgical. Laparoscopy is the most important procedure used to diagnose endometriosis as there are many other diseases that can cause similar symptoms as well as the target population in the present study was in early adulthood. Other symptoms were Painful bowel movement or defecation $6 \%$, followed by painful urination $2 \%$. Our results were inconsistent with a New Zealand study evaluating 163 patients with histological confirmation of endometriosis from 2003 to 2009. Authors concluded that the main complaint for patients with endometriosis was dysmenorrhea by 55\% among adults [25].[26] pointed that $78 \%$ out of 23 patient with endometriosis has non cyclic pelvic pain while in our study result indicated that non cyclic pelvic pain reflected on $26 \%$ of women with endometriosis.

The present study showed that post education knowledge scores was significantly higher than preeducation $(\mathrm{t}=-21.664, \mathrm{P}<0.001)$. This might be related to the effect of health education sessions that were given to women and how The finding was in agreement with [27] who reported that education is imperative for patients with suspected endometriosis. Armed with knowledge, women are better able to cope with the disease and make informed decisions regarding care. Nurse practitioners should educate patients regarding the condition's prevalence, pathogenesis, and known risk and protective factors. They should also discuss treatment options and their associated risks and benefits. Frequency of appointments will be determined by a woman's individual situation and needs. As well as the result was consistent with [28] who indicated that Knowledge development of theory can be challenging for experience deficits related to theoretical foundations, evaluation, or application. Women anxiety gave way to enthusiasm with the implementation of teaching and learning strategies based on adult learning theory.

The present study results indicated a positive correlation between pre-education type of diet and symptoms of endometriosis that was exaggerated with women who intake red meat rather than who intake fruits and vegetables $(\mathrm{r}=0.334, \mathrm{P}=0.015)$. This present study finding agree to a certain level with several studies find a strong connection between endometriosis symptoms and diets high in red meat and low in green vegetables and fresh fruit. This finding was congruent with the first scientific article that addresses the subject of type of diet and endometriosis was published in 2004, by [29]. They found that the highest weekly intake of fruits and vegetables was inversely associated with risk of developing the disease (vegetables: RR 0.3; 95CI 0.2-0.5, $\mathrm{p}=0.002$; and fruits: RR 0.6; 95\% CI 0.4-0.8, p=0.002). In contrast, consumption of red meat, cold cuts and sausages has been identified as a risk factor for the development of the disease. This fits with other studies finding similar connections between these eating patterns and endometrial cancer and fibroids (noncancerous tumors of the uterus). One study in this area compared healthy women and women with endometriosis, finding that women who eat beef every day were nearly twice as likely to have endometriosis, while those who got seven or more fruit and vegetable servings a week were at least $40 \%$ less likely[13]. Unfortunately, [14] proposed that, there has been very little research done to figure out if eating certain foods can help improve endometriosis symptoms. They also ask, if someone know that some young women who eat lots of fruits, vegetables, and healthy fats while limiting their intake of red meat and unhealthy fats are less likely to have endometriosis. Some young women with endometriosis say they feel better when they eat a nutritious diet and some experts believe that eating certain foods can help endometriosis symptoms by reducing inflammation and estrogen levels in the body. Even if eating nutritious food doesn't necessarily make your "endometriosis" symptoms better, there are lots of other benefits to a healthy diet [14].

Regarding exercise practices (physical activity), the present study did not report any significant correlation between post education exercise with severity of symptoms $(\mathrm{r}=0.052, \mathrm{p}=0.87)$, as well as, the researchers didn't get any information from women regarding their exercise practices before recruitment to education sessions and only $20 \%$ (10) from the total sample who accept to use exercise as a modality to reduce severity of pain related to endometriosis \& they did not maintain regular exercise all over the three months follow up, as they do in diet one. This might be related to our culture that doesn't tackle the importance of exercise for our health as well as sociodemographic standard of women that sometimes interfere with that issue. This result was supported by [30] who assessed individual factors that influenced the quality of life and the intensity of pain of patients with endometriosis. Their results demonstrated that the use of analgesics could be less effective in patients with endometriosis who exercised regularly. The authors did not point if in women with endometriosis, the pelvic pain was a limiting factor for exercising.

Also [15] indicated that healthy behavior is not only physical activity, but also a construct of mental, educational, and environmental situations that enables us to live a healthy life. In the situation of endometriosis and pain, or perception of pain, social factors and environment play an important role; thus, the social and psychological support might be of further relevance. Adding to the previous, a case control study, related the risk of endometriosis to other less studied factors such as anthropometric variables (height, weight and BMI), physical exercise, smoking, and alcohol consumption. The authors observed an inverse association between 
vigorous physical exercises and endometriosis, although without statistical significance. [15], also pointed that is possible that the protective effect of exercise in patients with endometriosis result from the fact that women who suffer from endometriosis do not feel well enough to practice exercise. In general, curative treatment alone is not enough to promote health. Supporting healthy behavior is the main goal of health promotion, and healthy behavior is a result of a multidimensional approach that is influenced by several factors [31].[32] stated that healthy behavior is not only physical activity, but also a construct of mental, educational, and environmental situations that enables us to live a healthy life. In the situation of endometriosis and physical activity, or perception of physical activity, social factors and environment play an important role; thus, the social and psychological support might be of further relevance.

\section{Conclusion}

The overall findings in the present study has indicated that health education sessions about changing lifestyle particularly dietary health education was an effective and had brought about significant improvement of knowledge and reduction of endometriosis related pain with substantial improvement of pain for three months after completion of health education.

\section{Recommendation}

- Health education of women to raise their awareness about endometriosis related factors should be a priority to ensure early diagnosis of the disease.

- A simplified and comprehensive booklet should be available for all patients with endometriosis. This booklet should include a clear, brief and simple explanation about non pharmacological modalities to reduce endometriosis related pain.

- The presence of nurse educator to improve patients' knowledge about importance of healthy lifestyle to reduce endometriosis related pain

- Further studies requested, in consideration of the fact that occupational or leisure exercise is strongly related with other determinants of endometriosis, such as economic status, reproductive pattern, and menstrual pattern.

- Replication of the study using a larger probability sample from different geographical areas to help for identifying the effect of changing lifestyle on endometriosis related pain.

\section{Limitation of the study:}

certain obstacles faced the researchers during the implementation of health education such as; $20 \%$ only from the study sample who agreed to use the exercises protocol plus the diet one, and the rationale of other $80 \%$ was as they have severe pain, fatigue and were not able to either walk by regular manner during the study period or using Gymnastic or running. So, the effect of exercise did not reflect significantly on their pain intensity.

\section{References}

[1]. K.E. Nnoaham, L. Hummelshoj , P. Webster, T. d'Hooghe , F. de Cicco Nardone ,C. de Cicco Nardone, C.Jenkinson , S.H.Kennedy, and K.T. Zondervan , Impact of endometriosis on quality of life and work productivity: a multicenter study across ten countries, Fertil Steril,96 (2) ,2011,366-373.

[2]. R.S.Schenken, Pathogenesis, Clinical Features, and Diagnosis of Endometriosis, 2013. http://www.uptodate.com/contents/pathogenesis-clinical-features-and-diagnosis-of endometriosis?

[3]. P. Vercellini, P. Viganò, E. Somigliana, and L. Fedele, Endometriosis: pathogenesis and treatment. Nat Rev Endocrinol, 10: 2014, 261-75.

[4]. P.A.Rogers, T.M. D'Hooghe, A.Fazleabas, C.E.Gargett, L.C. Giudice , G.W. Montgomery, L. Rombauts , L.A. Salamonsen, and K.T. Zondervan, Priorities for endometriosis research: recommendations from an international consensus workshop , Reprod Sci,16(4), 2009, 335-46

[5]. G.D. Adamson, S. Kennedy, and L. Hummelshoj, Creating solutions in endometriosis: global collaboration through the World Endometriosis Research Foundation, J of Endometriosis,2(1) 2010:3-6.

[6]. L.C.Giudice, Clinical practice. Endometriosis. N Engl J Med., 362(25), 2010, 2389-2398. [PMC free article][PubMed].

[7]. F.Parazzini, P.Viganò, M. Candiani, and L.Fedele, Diet and endometriosis risk: a literature review, Reproductive Bio-Medicine Online ,26, 2013, 323-336.doi: 10.1016/j.rbmo.

[8]. A.Chan, Endometriosis: Causes, Symptoms \& Treatments Clinical review. http://dx.doi.org/10.1136/bmj.g1752 (Published) Cite this as: BMJ, 2014,348: g1752.

[9]. P.Viganò, Endometriosis: Epidemiology and Etiological Factors. Best practice clinical obstetrics and gynaecology 18, 2012, 177200.

[10]. A. Koppan, The Impact Of Surgical Management On Quality Of Life Endometriosis, 2011, Faculty Of Health Science.

[11]. G. Halpern, G.Scho, and A. Kopelman, Nutritional aspects related to endometriosis, 2015, p 513.

[12]. C.M. Bonocher, M. L. Montenegro, J.C. Rosa e Silva, R.A. Ferriani, and J.Meola, Endometriosis and physical exercises: a systematic review. Reproductive Biology and Endocrinology; 12:4, 2014. doi: 10.1186/1477-7827-12-4.

[13]. Healthy Women, Lifestyle and Dietary Changes for Endometriosis, National Women's Health Resource Center, 2017, Inc. http://www.healthywomen.org/sites/default/files/endometriosis-lifestyle.jpg

[14]. Health Guides, Endometriosis: Nutrition and Exercise Posted under Health Guides. Updated 20 January 2017.

[15]. E.Ricci, P.Viganò, , S. Cipriani, F. Chiaffarino, S. Bianchi, G. Rebonato, and F. Parazzini, Physical activity and endometriosis risk in women with infertility or pain. Systematic review and meta-analysis, Medicine,95:40, 2016, (e4957). 
[16]. J.S. Berek, and E. Novak, gynecology ( Philadelphia, Wolters Kluwer Health/Lippincott Williams and Wilkins), 2012.

[17]. M. Freyd, A Graphic Rating Scale for Teachers. The Journal of Educational Research:Vol. 8, No. 1923, pp. 433-439.

[18]. C.Knop, M. Oeser, L. Bastian, U.Lange, M. Zdichavsky, and M. Blauth, Development And Validation of the Visual Analogue Scale, (VAS) score, 104(6), 2001, 1488-97.

[19]. M. Moradi, M. Parker, A. Sneddon, V. Lopez , and D. Ellwood, Impact of endometriosis on women's lives: a qualitative study. BMC Women's Health, 14:123,2014. mDOI: 10.1186/1472-6874-14-123.

[20]. I.M. Matalliotakis, H. Cakmak, Y.G. Fragouli, A.G. Goumenou, N.G. Mahutte, and A. Arici. Epidemiological characteristics in women with and without endometriosis in the Yale series. Arch Gynecol Obstet; 277, 2008, 389-393.

[21]. I. Flores, S. Abreu, S. Abac, J. Fourquet, J. Laboy, and C. Ri'os Bedoya. Self-reported prevalence of endometriosis and its symptoms among Puerto Rican women. Int J Gynecol Obstet, 100, 2008, 257-261.

[22]. H.M. Mamdouha, M.M. Mortadaa, I.F. Kharbousha, and H.,A. Abd-Elateefb, Epidemiologic determinants of endometriosis among Egyptian women: a hospital-based case-control study. Journal of the Egyptian Public Health Association, 86, 2011, 21-26.

[23]. E.B. Janssen, A.C. Rijkers, K. Hoppen brouwers, C. Meuleman, and T.M. D'Hooghe, Prevalence of endometriosis diagnosed by laparoscopy in adolescents with dysmenorrhea or chronic pelvic pain: a systematic review. Hum Reprod Update; 19(5),2013, 57082.

[24]. A. Ragab, M. Shams, A. Badawy, and M. A. Alsammani, Prevalence of endometriosis among adolescent school girls with severe dysmenorrhea: A cross sectional prospective study. International Journal of Health Sciences, Qassim University, Vol. 9, No. 3,2015 .

[25]. S. Abbas, P. Ihle, I. Koster, and I .Schubert, Prevalence and incidence of diagnosed endometriosis and risk of endometriosis in patients with endometriosis-related symptoms: findings from a statutory health insurance-based cohort in Germany. Eur J Obstet Gynecol Reprod Biol; 160 ,(1), 2012,79-83.

[26]. M. Api, A. T. Boza, S. Kayatas, and M. Eroglu, Effect of Surgical Removal of Endometriomas on Cyclic and Non-cyclic Pelvic Pain. Royan Institute, International Journal of Fertility and Sterility; 9, 2, 2015, Pages: 183-188

[27]. G.Altman, and M. Wolcyzk, Endometriosis: Overview and Recommendations for Primary Care Nurse Practitioners. The Journal for Nurse Practitioners ; Volume 6, Issue 6, 2010, 427-434.

[28]. D.E.McMillan, S. Bell, E.E. Benson, L.L. Mandzuk , D.M. Matias, M.J. McIvor, J.E. Robertson, K.L. Wilkins, From anxiety to enthusiasm: facilitating graduate nursing students' knowledge development in science and theory. Nurs Educ.; 46(2),2007, 88-91.

[29]. F. Parazzini, F. Chiaffarino, M. Surace, L. Chatenoud, S. Cipriani, V.Chiantera, G.Benzi and L.Fedele, Selected food intake and risk of endometriosis. Hum Reprod., 19, (8), 2004,1755-9.

[30]. Koppan A, Hamori J, Vranics I, Garai J, Kriszbacher I, Bodis J, Rebek-Nagy G, Koppan M. Pelvic pain in endometriosis: painkillers or sport to alleviate symptoms? Acta Physiol Hung. 2010;97:234-239. doi: 10.1556/APhysiol.97.2010.2.10.

[31]. R. Leischik, B. Dworrak, M. Strauss, B. Przybylek, T. Dworrak, D. Schöne, M. Horlitz, and A. Mügge, Plasticity of health. German J Med.,1, 2016, 1-17.

[32]. C. Fiuza-Luces, N. Garatachea, N.A. Berger, and A Lucia, Exercise is the real polypill. Physiology, 28, 2013, 330-58. 